\title{
CORRESPONDENCE
}

\section{Smoking cessation: a critical investigative tool in COPD}

\section{To the Editors:}

The recent longitudinal assessment of airway inflammation in chronic obstructive pulmonary disease (COPD) patients, before and after 1-yr smoking cessation [1], is a perfect example of what clinical researchers should do in order to expand the current knowledge base in the area of smoke-related disorders. As IND [2] appropriately points out in his ancillary editorial to the previously mentioned paper, the findings are not conclusive due to the lack of study controls, together with the small number of COPD subjects studied.

Nevertheless, the effect of smoking cessation on COPD is an important topic and further efforts should be devoted towards high-quality longitudinal studies of smoking cessation. International guidelines for COPD (British Thoracic Society, American Thoracic Society, Global Initiative for Chronic Obstructive Lung Disease) recommend smoking cessation interventions for smokers with COPD that are equivalent to interventions for healthy smokers. However, the available evidence in the medical literature indicates that, in disagreement with smokers in the general population, COPD patients who smoke typically respond poorly to smoking cessation efforts for a number of reasons. They have a greater degree of physical nicotine dependence [3, 4] and appear to be less motivated in relation to quitting smoking [5, 6]. Furthermore, patients with COPD have repeatedly been characterised as a population of chronically ill patients with a higher than normal prevalence of psychiatric disorders such as depression [7, 8]. It is well known that for smokers with depression, or a history of depression, it is far more difficult to quit smoking.

Hence, it is somewhat surprising to note that the 1-yr smoking cessation rate of $\sim 50 \%$ achieved in a population of COPD outpatients by these authors' counselling programme is one of the highest ever reported in smoking cessation literature. This is outstanding in consideration of the fact that this result was accomplished without the support of recommended cessation treatments (such as bupropion or nicotine replacement therapy), which are known to appreciably improve quit rates even in patients with COPD [9]. In consideration of this, the authors' decision to study only 28 COPD smokers in this intervention trial appears not to be supported by the logic of powering the sample size for the current study, in that the excellent outcomes rates of their smoking cessation programme could not be predicted in COPD patients.

Smoking cessation is a dynamic process and full cessation is typically obtained after a few attempts; for this reason, a rescue component for relapse is generally integrated into most smoking cessation programmes. There is little information about this as it seems that full cessation was attained in all quitters at 2 months and sustained, thereafter, until conclusion of the 1-yr smoking cessation trial with no documented withdrawals or late relapsing.

Finally, drop-outs from smoking cessation trials are common (attrition rates of $\sim 20-50 \%$ are generally reported). In the present study, it appeared that all participants (this includes not only relapsers/continuing smokers, but also quitters) completed all time points in the study. This is most unusual and requires an explanation.

High quit rates would be desirable in a population that generally respond poorly to smoking cessation efforts, so as to simplify recruitments into research studies of chronic obstructive pulmonary disease smoking cessation and to make the most of healthcare resources. Hence, it is crucial to know more details about recruitment modalities, patients' characteristics (particularly in terms of social, economical and demographical factors), incentive plans and intervention schemes of the smoking cessation programme used in the study. Perhaps this would be the first time that evidence with regard to smoking cessation interventions that are specifically developed for smokers with chronic obstructive pulmonary disease is provided.

\section{R. Polosa}

Dipartimento di Medicina Interna e Specialistica, Università di Catania, Catania, Italy.

\section{REFERENCES}

1 Willemse BW, ten Hacken NH, Rutgers B, Lesman-Leegte IG, Postma DS, Timens W. Effect of 1-year smoking cessation on airway inflammation in COPD and asymptomatic smokers. Eur Respir J 2005; 26: 835-845.

2 Ind PW. COPD disease progression and airway inflammation: uncoupled by smoking cessation. Eur Respir J 2005; 26: 764-766.

3 Sach K, Hall R, Sachs B. Success of rapid smoking therapy in smokers with pulmonary and coronary heart diseases. Am Rev Respir Dis 1981; 123: 111-116.

4 Jimenez-Ruiz CA, Masa F, Miravitlles M, et al. Smoking characteristics: differences in attitudes and dependence between healthy smokers and smokers with COPD. Chest 2001; 119: 1365-1370.

5 Walters N, Coleman T. Comparison of the smoking behaviour and attitudes of smokers who attribute respiratory symptoms to smoking with those who do not. Br J Gen Pract 2002; 52: 132-134.

6 Clark MA, Hogan JW, Kviz FJ, Prohaska TR. Age and the role of symptomatology in readiness to quit smoking. Addictive Behav 1999; 24: 1-16.

7 Dudley DL, Glaser EM, Jorgenson BN, et al. Psychosocial concomitants to rehabilitation in chronic obstructive 
pulmonary disease. Part I. Psychosocial and psychological considerations. Chest 1980; 77: 413-420.

8 Isoaho R, Keistinen T, Laippala P, Kivela SL. Chronic obstructive pulmonary disease and symptoms related to depression in elderly persons. Psychol Rep 1998; 76: 287-297.

9 van der Meer RM, Wagena EJ, Ostelo RW, Jacobs JE, van Schayck CP. Smoking cessation for chronic obstructive pulmonary disease. Cochrane Database Syst Rev 2003; 2 CD002999.

DOI: $10.1183 / 09031936.06 .00145805$

\section{From the authors:}

We appreciate the comments by $\mathrm{R}$. Polosa regarding our recent article in the European Respiratory Journal [1]. In this study, we described unexpectedly high quit rates, both for asymptomatic smokers and smokers with chronic obstructive pulmonary disease (COPD). Since smoking cessation is a difficult task, especially in COPD patients, R. Polosa's most important question is how we obtained such high quit rates, i.e. which smoking-cessation programme was used and what were the subjects' characteristics. Furthermore, he questioned whether our study had the power to raise any tools to develop a special smoking-cessation programme for COPD patients. We are pleased to have the opportunity to give more information about our experiences on smoking cessation.

In our study, we used an intensive group-orientated smokingcessation programme, which has been described and commented on in more detail elsewhere [2]. In short, the programme is based on cognitive behavioural therapy and relapse prevention according to the model of Marlatt [3]. It consisted of 15 group meetings during $1 \mathrm{yr}, 10$ meetings within the first 3 months and another five throughout the rest of the year. In addition, the participants attended seven hospital visits before the smoking-cessation programme started, and three hospital visits throughout the rest of the year (at 2, 6 and 12 months).

We included patients with COPD and asymptomatic smokers who were willing to quit smoking and to undergo several tests, including two bronchoscopies. Participants were recruited by newspaper advertisements and at our outpatient clinic. Participants originated from the north of the Netherlands (rural and urban areas). Their motivations to quit were: 1) to improve health; 2) to become independent of cigarettes; 3) not to be a social outcast; and 4) not to smoke around grandchildren. Most individuals had tried to quit between one to three times previously.

We offered our patients nicotine-replacement therapy (NRT), yet none of those patients who had quit for $1 \mathrm{yr}$ had used NRT. Only three participants (two asymptomatic smokers and one smoker with COPD) tried NRT for 3-14 days; however, none of them succeeded in smoking cessation. Bupropion was not used in this study simply because the study started in 1998 and bupropion was not introduced into the Netherlands until 2000. In our opinion, the high quit rates are probably due to the intensive smoking-cessation course in combination with our research setting.
As soon as a smoker started to smoke again, we had to withdraw them from the study, since the medical ethics committee decided it was unethical to re-assess individuals who had relapsed after one smoking-cessation attempt. No participant who successfully quit smoking for $1 \mathrm{yr}$ dropped out of the study. Our explanation for the relative lack of dropouts is the high state of motivation of the participants, together with intensive guidance in the smoking-cessation programme. As we stated in our article, all 12 COPD quitters produced sufficient sputum samples at the four visits, but the bronchial biopsy of one patient was of insufficient quality. Of the 16 asymptomatic smokers, 15 produced good-quality sputum samples at all time points, and 14 out of the 16 had biopsies of good quality at all time points.

Only 28 smokers with COPD were included in this study and 12 of them succeeded in quitting smoking for $1 \mathrm{yr}$. Only these patients were included in the analyses. Of course, we would have liked to have included more COPD patients; however, we were limited in time, money and, unfortunately, in patients with COPD who did not use inhaled or oral corticosteroids.

We agree with R. Polosa that smokers with COPD may need a different approach towards smoking-cessation guidance than smokers without COPD, since they have greater nicotine dependence and a higher prevalence of depression-like symptoms. In our study, we did not find a significant difference in quit rates between asymptomatic smokers $(68 \%)$ and smokers with COPD (42\%) [2]. Our study was not set up to evaluate the smoking-cessation programme used and our population was too small to generalise the results. Nevertheless, the results are the most promising, given the $50 \%$ abstinence! Despite similar success rates in asymptomatic smokers and COPD patients, it was remarkable that, from the unsuccessful individuals, most COPD patients $(37 \%)$ relapsed within the first 2 months of smoking cessation, whereas most asymptomatic smokers $(20 \%)$ did so between 2 and 6 months [2].

We hypothesise that special smoking-cessation guidance for chronic obstructive pulmonary disease patients may increase quit rates in this group of smokers. Therefore, we propose a combination of intensive 1-yr cognitive behavioural therapy, relapse prevention and the use of pharmacological support during the first 2 months. In our opinion, a research setting will contribute to higher quit rates.

\section{B.W.M. Willemse ${ }^{*, \#}$, N.H.T. ten Hacken ${ }^{\#}$, D.S. Postma ${ }^{\#}$ and W. Timens*}

Depts of *Pathology, and \#Pulmonology, University Medical Center Groningen, University of Groningen, Groningen, The Netherlands.

\section{REFERENCES}

1 Willemse BWM, ten Hacken NHT, Rutgers B, LesmanLeegte IGAT, Postma DS, Timens W. Effect of 1-year smoking cessation on airway inflammation in COPD 